Supporting Information for the World Wide Web Edition

\title{
A General Framework for Development and Data Analysis of Competitive High-Throughput Screens for Small-Molecule Inhibitors of Protein-Protein Interactions by Fluorescence Polarization
}

Michael H. A. Roehrl, Julia Y. Wang, and Gerhard Wagner

The derivation of equations in this paper can be readily accomplished with Mathematica

(Wolfram Research, Champaign, IL). The following shows input and raw output from Mathematica 4.1.

Equation [5]

Input:

Simplify $[$ Solve $[\{\mathrm{KD} 1=\mathrm{R} * \mathrm{LS} / \mathrm{RLS}, \mathrm{RT}=\mathrm{R}+\mathrm{RLS}, \mathrm{LST}=\mathrm{LS}+\mathrm{RLS}, \mathrm{FSB}=1-\mathrm{LS} / \mathrm{LST}\}, \mathrm{RT},\{\mathrm{R}, \mathrm{LS}, \mathrm{RLS}\}]]$

Output:

$\left\{\mathrm{RT} \rightarrow \frac{\mathrm{FSB}(-\mathrm{KD} 1-\mathrm{LST}+\mathrm{FSB} \mathrm{LST})}{-1+\mathrm{FSB}}\right\}$

Equation [6]

Input:

Simplify [Solve $[\{\mathrm{KD} 1==\mathrm{R} * \mathrm{LS} / \mathrm{RLS}, \mathrm{RT}=\mathrm{R}+\mathrm{RLS}, \mathrm{LST}=\mathrm{LS}+\mathrm{RLS}, \mathrm{FSB}==1-\mathrm{LS} / \mathrm{LST}\}, \mathrm{FSB},\{\mathrm{R}, \mathrm{LS}, \mathrm{RLS}\}]$ ] 


\section{Output:}

$$
\begin{aligned}
& \left\{\mathrm{FSB} \rightarrow \frac{\mathrm{KD} 1+\mathrm{LST}+\mathrm{RT}-\sqrt{-4 \mathrm{LSTRT}+(\mathrm{KD} 1+\mathrm{LST}+\mathrm{RT})^{2}}}{2 \mathrm{LST}}\right\}, \\
& \left\{\mathrm{FSB} \rightarrow \frac{\mathrm{KD} 1+\mathrm{LST}+\mathrm{RT}+\sqrt{-4 \mathrm{LSTRT}+(\mathrm{KD} 1+\mathrm{LST}+\mathrm{RT})^{2}}}{2 \mathrm{LST}}\right\}
\end{aligned}
$$

(Note that only the first root has physical meaning.)

\section{Equation [8]}

Input:

Simplify $[$ Solve $[\{\mathrm{KD} 1=\mathrm{R} * \mathrm{LS} / \mathrm{RLS}, \mathrm{RT}=\mathrm{R}+\mathrm{RLS}, \mathrm{LST}=\mathrm{LS}+\mathrm{RLS}+\mathrm{NS} * \mathrm{LS}, \mathrm{FSB}=1-\mathrm{LS} / \mathrm{LST}\}, \mathrm{RT},\{\mathrm{R}, \mathrm{LS}, \mathrm{RLS}\}]$

Output:

$$
\left\{\mathrm{RT} \rightarrow-\frac{(\mathrm{KD} 1+\mathrm{LST}-\mathrm{FSB} \mathrm{LST})(\mathrm{FSB}-\mathrm{NS}+\mathrm{FSB} \mathrm{NS})}{-1+\mathrm{FSB}}\right\}
$$

\section{Equation [9]}

Input:

Simplify $[$ Solve $[\{\mathrm{KD} 1=\mathrm{R} * \mathrm{LS} / \mathrm{RLS}, \mathrm{RT}=\mathrm{R}+\mathrm{RLS}, \mathrm{LST}=\mathrm{LS}+\mathrm{RLS}+\mathrm{NS}$ * LS, FSB == $1-\mathrm{LS} / \mathrm{LST}\}, \mathrm{FSB},\{\mathrm{R}, \mathrm{LS}, \mathrm{RLS}\}]$ ]

Output:

$$
\begin{aligned}
& \left\{\mathrm{FSB} \rightarrow \frac{\mathrm{KD} 1+\mathrm{LST}+\mathrm{KD} 1 \mathrm{NS}+2 \mathrm{LST} \mathrm{NS}+\mathrm{RT}+\sqrt{\mathrm{KD}^{2}(1+\mathrm{NS})^{2}+(\mathrm{LST}-\mathrm{RT})^{2}+2 \mathrm{KD} 1(1+\mathrm{NS})(\mathrm{LST}+\mathrm{RT})}}{2 \mathrm{LST}(1+\mathrm{NS})}\right\} \\
& \left\{\mathrm{FSB} \rightarrow \frac{\mathrm{KD} 1+\mathrm{LST}+\mathrm{KD} 1 \mathrm{NS}+2 \mathrm{LST} \mathrm{NS}+\mathrm{RT}-\sqrt{\mathrm{KD} 1^{2}(1+\mathrm{NS})^{2}+(\mathrm{LST}-\mathrm{RT})^{2}+2 \mathrm{KD} 1(1+\mathrm{NS})(\mathrm{LST}+\mathrm{RT})}}{2 \mathrm{LST}(1+\mathrm{NS})}\right\}
\end{aligned}
$$

(Note that only the second root has physical meaning.) 


\section{Equation [12]}

Input:

Simplify [Solve $[\{\mathrm{KD} 1 \mathrm{~A}=\mathrm{R} * \mathrm{LS} / \mathrm{RLS}, \mathrm{RT}=\mathrm{R}+\mathrm{RLS}, \mathrm{LST}=\mathrm{LS}+\mathrm{RLS}, \mathrm{FSBA}=1-\mathrm{LS} / \mathrm{LST}, \mathrm{FSBA}==(1+\mathrm{NS}) * \mathrm{FSB}-\mathrm{NS}\}$, RT, \{FSBA, R, LS, RLS \} ] ]

Output:

$$
\left\{\mathrm{RT} \rightarrow \frac{(\mathrm{FSB}-\mathrm{NS}+\mathrm{FSBNS})(-\mathrm{KD} 1 \mathrm{~A}+(-1+\mathrm{FSB}) \mathrm{LST}(1+\mathrm{NS}))}{(-1+\mathrm{FSB})(1+\mathrm{NS})}\right\}
$$

\section{Equation [16]}

Input:

Simplify [Solve [ $\{\mathrm{KD} 1=\mathrm{R}$ * LS / RLS, KD2 =s R $\mathrm{L} / \mathrm{RL}, \mathrm{RT}=\mathrm{R}+\mathrm{RLS}+\mathrm{RL}, \mathrm{LST}=\mathrm{LS}+\mathrm{RLS}, \mathrm{LT}=\mathrm{L}+\mathrm{RL}, \mathrm{FSB}==1-\mathrm{LS} / \mathrm{LST}\}, \mathrm{LT}$, $\{R, L S, R L S, L, R L\}]\}$

$\underline{\text { Output: }}$

$$
\left\{\mathrm{LT} \rightarrow-\frac{(\mathrm{FSB}(\mathrm{KD} 1-\mathrm{KD} 2)+\mathrm{KD} 2)\left(\mathrm{FSB}^{2} \mathrm{LST}+\mathrm{RT}-\mathrm{FSB}(\mathrm{KD} 1+\mathrm{LST}+\mathrm{RT})\right)}{(-1+\mathrm{FSB}) \mathrm{FSB} \mathrm{KD1}}\right\}
$$

\section{Equation [19]}

Input:

Simplify [Solve [ $\{\mathrm{KD} 1=\mathrm{R} * \mathrm{LS} / \mathrm{RLS}, \mathrm{KD} 2=\mathrm{R} * \mathrm{~L} / \mathrm{RL}, \mathrm{RT}=\mathrm{R}+\mathrm{RLS}+\mathrm{RL}, \mathrm{LST}=\mathrm{LS}+\mathrm{RLS}, \mathrm{LT}=\mathrm{L}+\mathrm{RL}+\mathrm{N} * \mathrm{~L}$, FSB $==1-\mathrm{LS} / \mathrm{LST}\}, \mathrm{LT},\{\mathrm{R}, \mathrm{LS}, \mathrm{RLS}, \mathrm{L}, \mathrm{RL}\}]]$

Output:

$$
\left\{\mathrm{LT} \rightarrow-\frac{(\mathrm{KD} 2(1+\mathrm{N})+\mathrm{FSB}(\mathrm{KD} 1-\mathrm{KD} 2(1+\mathrm{N})))\left(\mathrm{FSB}^{2} \mathrm{LST}+\mathrm{RT}-\mathrm{FSB}(\mathrm{KD} 1+\mathrm{LST}+\mathrm{RT})\right)}{(-1+\mathrm{FSB}) \mathrm{FSB} \mathrm{KD} 1}\right\}
$$

\section{Equation [27]}

Input:

Simplify [Solve [ $\{\mathrm{KD} 1=\mathrm{R} * \mathrm{LS} / \mathrm{RLS}, \mathrm{KD} 2=\mathrm{R} * \mathrm{~L} / \mathrm{RL}, \mathrm{KD} 3=\mathrm{RL} * \mathrm{LS} / \mathrm{RLLS}, \mathrm{KD} 2 * \mathrm{KD} 3$ / KD1 == RLS * L / RLLS,

$R T==R+R L S+R L+R L S, L S T==L S+R L S+R L L S, L T==L+R L+R L L S, F S B=1-I S / L S T\}, L T,\{R, L S, R L S, L, R L, R L L S\}$ ] 


\section{Output:}

$$
\begin{gathered}
\left\{\mathrm{LT} \rightarrow-\left(( \mathrm { FSB } ^ { 2 } \mathrm { LST } + \mathrm { RT } - \mathrm { FSB } ( \mathrm { KD } 1 + \mathrm { LST } + \mathrm { RT } ) ) \left(-(-1+\mathrm{FSB}) \mathrm{KD} 2 \mathrm{KD}^{2}+\mathrm{KD} 1\right.\right.\right. \\
\left.\left.\quad\left((-1+\mathrm{FSB}) \mathrm{KD} 2 \mathrm{KD} 3+(\mathrm{KD} 3+\mathrm{LST}-\mathrm{FSB} \mathrm{LST})\left(-\mathrm{FSB}^{2} \mathrm{LST}-\mathrm{RT}+\mathrm{FSB}(\mathrm{KD} 3+\mathrm{LST}+\mathrm{RT})\right)\right)\right)\right) / \\
\left.\left((-1+\mathrm{FSB}) \mathrm{KD} 1(\mathrm{KD} 1-\mathrm{KD} 3)\left(\mathrm{FSB}^{2} \mathrm{LST}+\mathrm{RT}-\mathrm{FSB}(\mathrm{KD} 3+\mathrm{LST}+\mathrm{RT})\right)\right)\right\}
\end{gathered}
$$

\section{Equation [30]}

Input:

Simplify [Solve $[\{\mathrm{KD} 1=\mathrm{R} * \mathrm{LS} / \mathrm{RLS}, \mathrm{KD} 2=\mathrm{R} * \mathrm{~L} / \mathrm{RL}, \mathrm{KD} 3=\mathrm{RL} * \mathrm{LS} / \mathrm{RLLS}, \mathrm{KD} 2$ * KD3 / KD1 =: RLS *L/RLLS,

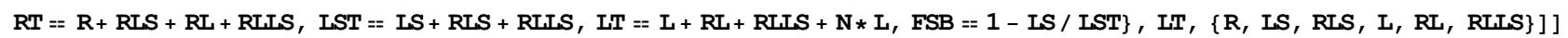

\section{Output:}

$$
\begin{gathered}
\left\{\mathrm{LT} \rightarrow-\left(\left(\mathrm{FSB}^{2} \mathrm{LST}+\mathrm{RT}-\mathrm{FSB}(\mathrm{KD} 1+\mathrm{LST}+\mathrm{RT})\right)\right.\right. \\
\quad\left(-(-1+\mathrm{FSB}) \mathrm{KD} 2 \mathrm{KD}^{2}(1+\mathrm{N})+\mathrm{KD} 1((-1+\mathrm{FSB}) \mathrm{KD} 2 \mathrm{KD} 3(1+\mathrm{N})+\right. \\
\left.\left.\left.(\mathrm{KD} 3+\mathrm{LST}-\mathrm{FSB} \mathrm{LST})\left(-\mathrm{FSB}^{2} \mathrm{LST}-\mathrm{RT}+\mathrm{FSB}(\mathrm{KD} 3+\mathrm{LST}+\mathrm{RT})\right)\right)\right)\right) / \\
\left.\left((-1+\mathrm{FSB}) \mathrm{KD} 1(\mathrm{KD} 1-\mathrm{KD} 3)\left(\mathrm{FSB}^{2} \mathrm{LST}+\mathrm{RT}-\mathrm{FSB}(\mathrm{KD} 3+\mathrm{LST}+\mathrm{RT})\right)\right)\right\}
\end{gathered}
$$

\section{Equation [43]}

Input:

Simplify [Solve $[((\mathrm{KD} 1-\mathrm{KD} 2)$ * FSB + KD2) * (LST * FSB^2 - (KD1 + LST + RT) * FSB + RT) / ( 1 - FSB $)$ * FSB * KD1) == LT, RT] ]

Output:

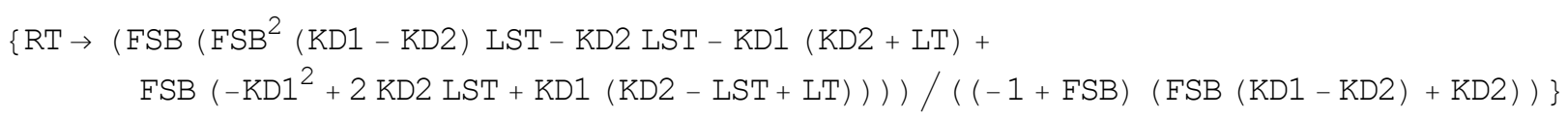

\section{Equation [44]}

Input:

Simplify $[D[((\mathrm{KD} 1-\mathrm{KD} 2)$ * FSB + KD2 $)$ * (LST * FSB^2 - (KD1 + LST + RT) * FSB + RT) / ( $1-\mathrm{FSB})$ * FSB * KD1), FSB] ] 


\section{Output:}

$$
\begin{aligned}
& \frac{1}{(-1+\mathrm{FSB})^{2} \mathrm{FSB}^{2} \mathrm{KD} 1}\left(2 \mathrm{FSB}^{3}(\mathrm{KD} 1-\mathrm{KD} 2) \mathrm{LST}+\right. \\
& \left.\mathrm{FSB}^{4}(-\mathrm{KD} 1+\mathrm{KD} 2) \mathrm{LST}-\mathrm{KD} 2 \mathrm{RT}+2 \mathrm{FSB} \mathrm{KD} 2 \mathrm{RT}-\mathrm{FSB}^{2}\left(\mathrm{KD}^{2}+\mathrm{KD} 1 \mathrm{LST}+\mathrm{KD} 2(-\mathrm{LST}+\mathrm{RT})\right)\right)
\end{aligned}
$$

Input:

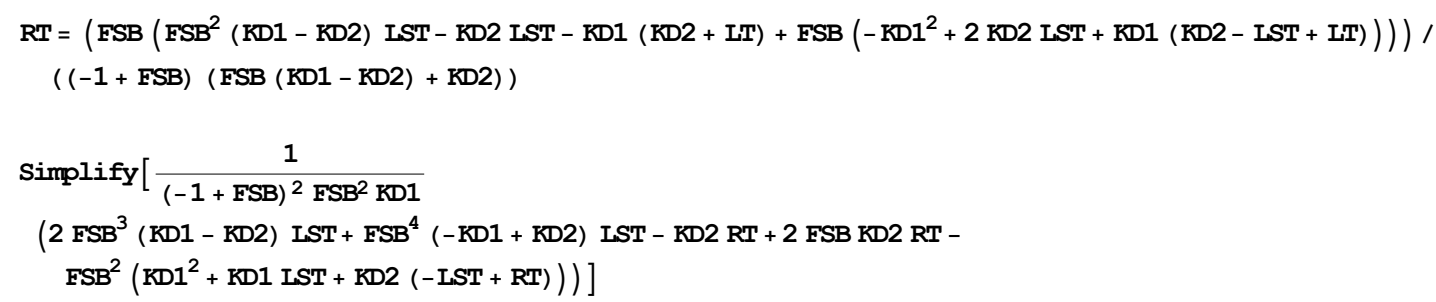

Output:

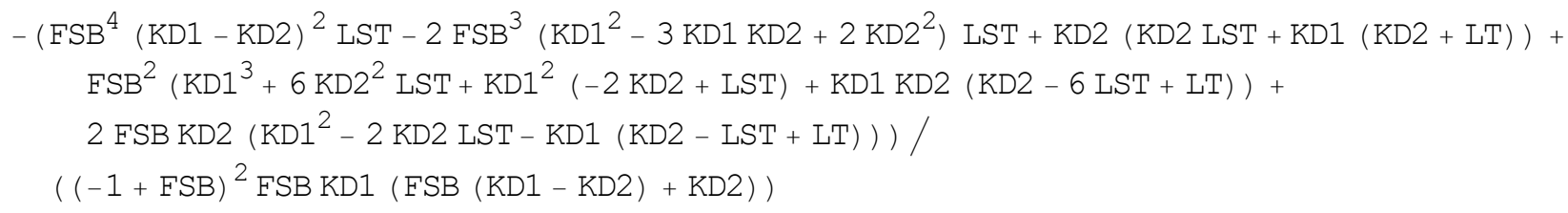

\section{Equation [46]}

Input:

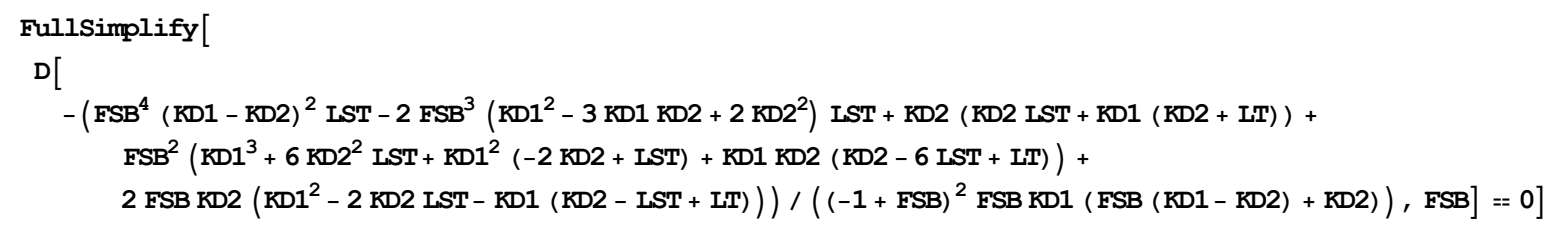

Output:

$$
\begin{gathered}
\left((\mathrm{FSB}(\mathrm{KD} 1-\mathrm{KD} 2)+\mathrm{KD} 2)^{2}\left(\mathrm{KD} 1\left(2 \mathrm{FSB}^{2}(\mathrm{KD} 1-\mathrm{KD} 2)-\mathrm{KD} 2+3 \mathrm{FSB} \mathrm{KD} 2\right)+(-1+\mathrm{FSB})^{3} \mathrm{KD} 2 \mathrm{LST}\right)+\right. \\
\left.(-1+\mathrm{FSB})^{3} \mathrm{KD} 1 \mathrm{KD} 2(2 \mathrm{FSB}(\mathrm{KD} 1-\mathrm{KD} 2)+\mathrm{KD} 2) \mathrm{LT}\right) / \\
\left((-1+\mathrm{FSB})^{3} \mathrm{FSB}^{2} \mathrm{KD} 1(\mathrm{FSB}(\mathrm{KD} 1-\mathrm{KD} 2)+\mathrm{KD} 2)^{2}\right)==0
\end{gathered}
$$

(Note that a necessary condition is that the numerator equals zero. It can be shown that numerator and denominator cannot be simultaneously zero under the physical restraints imposed on the variables.) 


\section{Equation [51]}

Input:

Simplify [Solve $[\{\mathrm{KD} 1=\mathrm{R} * \mathrm{LS} / \mathrm{RLS}, \mathrm{KD} 2=\mathrm{R} * \mathrm{~L} / \mathrm{RL}, \mathrm{RT}=\mathrm{R}+\mathrm{RLS}+\mathrm{RL}, \mathrm{LST}=\mathrm{LS}+\mathrm{RLS}, \mathrm{LT}=\mathrm{L}+\mathrm{RL}, \mathrm{FSB}==1-\mathrm{LS} / \mathrm{LST}\}, \mathrm{KD} 2$, $\{R, L S, R L S, L, R L\}]\}$

Output:

$$
\left\{\mathrm{KD} 2 \rightarrow \frac{\mathrm{FSB} \mathrm{KD} 1\left(\mathrm{FSB}^{2} \mathrm{LST}-\mathrm{LT}+\mathrm{RT}-\mathrm{FSB}(\mathrm{KD} 1+\mathrm{LST}-\mathrm{LT}+\mathrm{RT})\right)}{(-1+\mathrm{FSB})\left(\mathrm{FSB}^{2} \mathrm{LST}+\mathrm{RT}-\mathrm{FSB}(\mathrm{KD} 1+\mathrm{LST}+\mathrm{RT})\right)}\right\}
$$

Input:

Simplify $\left[\right.$ Solve $\left.\left[\left\{\frac{\mathrm{KD} 1+\mathrm{LST}+\mathrm{RT}-\sqrt{-4 \mathrm{LST} R T+(\mathrm{KD} 1+\mathrm{LST}+\mathrm{RT})^{2}}}{2 \mathrm{LST}}=(\mathrm{AU}-\mathrm{AF}) /((\mathrm{AB}-\mathrm{AU}) * \mathrm{Q}+\mathrm{AU}-\mathrm{AF})\right\}, \mathrm{AB}\right]\right]$

\section{Output:}

$$
\begin{aligned}
\{A B \rightarrow & \left(A F\left(-K D 1+L S T-R T+\sqrt{-4 L S T R T+(K D 1+L S T+R T)^{2}}\right)+\right. \\
& \left.A U\left(K D 1-K D 1 Q-L S T(1+Q)+(-1+Q)\left(-R T+\sqrt{-4 L S T R T+(K D 1+L S T+R T)^{2}}\right)\right)\right) / \\
& \left.\left(Q\left(K D 1+L S T+R T-\sqrt{-4 L S T R T+(K D 1+L S T+R T)^{2}}\right)\right)\right\}
\end{aligned}
$$

Input:

$\mathrm{AB}=$

$-\left(\mathrm{AF}\left(-\mathrm{KD} 1+\mathrm{LST}-\mathrm{RT}+\sqrt{-4 \mathrm{LST} R T+(\mathrm{KD} 1+\mathrm{LST}+\mathrm{RT})^{2}}\right)+\right.$ $\left.\mathrm{AU}\left(\mathrm{KD} 1-\mathrm{KD} 1 \mathrm{Q}-\mathrm{LST}(1+Q)+(-1+Q)\left(-\mathrm{RT}+\sqrt{-4 \mathrm{LST} \mathrm{RT}+(\mathrm{KD} 1+\mathrm{LST}+\mathrm{RT})^{2}}\right)\right)\right) /$

$\left(\mathrm{Q}\left(\mathrm{KD} 1+\mathrm{LST}+\mathrm{RT}-\sqrt{-4 \mathrm{LST} R T+(\mathrm{KD} 1+\mathrm{LST}+\mathrm{RT})^{2}}\right)\right)$

FullSimplify $[(A O B S-A F) /((A B-A O B S) * Q+A O B S-A F)]$

Output:

$$
\begin{aligned}
& (2(\mathrm{AF}-\mathrm{AOBS}) \mathrm{RT}) /\left(2 \mathrm{AOBS}(-1+\mathrm{Q}) \mathrm{RT}+\mathrm{AF}\left(\mathrm{KD} 1+\mathrm{LST}+\mathrm{RT}+\sqrt{-4 \mathrm{LSTRT}+(\mathrm{KD} 1+\mathrm{LST}+\mathrm{RT})^{2}}\right)-\right. \\
& \left.\mathrm{AU}\left(\mathrm{KD} 1+\mathrm{LST}-\mathrm{RT}+2 \mathrm{QRT}+\sqrt{-4 \mathrm{LSTRT}+(\mathrm{KD} 1+\mathrm{LST}+\mathrm{RT})^{2}}\right)\right)
\end{aligned}
$$

Input:

$Q=1$

FullSimplify $[(\mathrm{AOBS}-\mathrm{AF}) /((\mathrm{AB}-\mathrm{AOBS}) * Q+\mathrm{AOBS}-\mathrm{AF})]$ 


\section{Output:}

$\frac{(\mathrm{AF}-\mathrm{AOBS})\left(\mathrm{KD} 1+\mathrm{LST}+\mathrm{RT}-\sqrt{-4 \mathrm{LST} R T+(\mathrm{KD} 1+\mathrm{LST}+\mathrm{RT})^{2}}\right)}{2(\mathrm{AF} \mathrm{LST}-\mathrm{AU} \mathrm{LST})}$

